\title{
Through-The-Earth (TTE) Communications for Underground Mines
}

\author{
Josua Peña Carreño, Lucas Sousa e Silva, Sávio Oliveira de Almeida Neves, Leonardo Aguayo, Adoniran Judson \\ Braga, André Noll Barreto, and Luis Guilherme Uzeda Garcia
}

\begin{abstract}
The communication needs for rescue operations of trapped miners has recently motivated the creation of throughthe-earth communication systems, which are also envisaged for enabling autonomous mining operations. Because of the large attenuation of radio frequency waves in this propagation medium, these systems usually employ transmission through magnetic induction using very low frequencies. In this paper we present an overview of the possible applications but also of the technical hurdles faced in this type of communications system. We present a model of a TTE transmission system and discuss some modulation and digital transmission approaches for this medium.
\end{abstract}

Index Terms-Through-The-Earth Communications, Extreme Conditions Communications.

\section{INTRODUCTION}

The importance of communications systems in the mining industry has grown considerably in the past few years. Initially, the availability of communication links was a requirement for keeping in touch with miners, particularly in emergency situations. Communications were limited to voice and narrowband dispatch systems, but, more recently, there is also an increasing interest in autonomous mining equipment, which will require reliable wireless communications for operation. In this paper we are particularly concerned with communications for underground mines, which pose higher risks, and are characterized by a difficult communications scenario. Among possible applications for communications in underground mines we can cite: mining equipment automation; rock blasting; multimedia communication between miners; mine video surveillance; monitoring of miners' vital signs; detection of dangerous gases, and real-time monitoring and supervision of equipment and personnel.

One solution for communications in underground mines is to lay a traditional communication infrastructure, consisting of cables, fibers, and wireless repeaters. This however has a few disadvantages. Firstly, it requires a large and constant investment, particularly if one bears in mind the highly changeable mining environment, with new shafts and tunnels being dug

Josua Peña Carreño, Lucas Sousa e Silva, Sávio Oliveira de Almeida Neves, Leonardo Aguayo, Adoniran Judson Braga and André Noll Barreto are with the Microwave and Wireless System Laboratory (MWSL), Department of Electric Engineering, University of Brasília, Brasília, DF, Brazil. e-mails: josua.d.pena@ieee.org, lucassilva@aluno.unb.br, savio.oneves@aluno.unb.br, aguayo@unb.br, jbraga@ene.unb.br, andrebarreto@ene.unb.br

Luis Guilherme Uzeda Garcia is with Vale Technology Institute, Ouro Preto, MG, Brazil. e-mail: luis.uzeda@itv.org

The Associate Editor coordinating the review of this manuscript and approving it for publication was Prof. Lisandro Lovisolo.

Digital Object Identiïñę (DOI): 10.14209/jcis.2016.15 constantly. Secondly, this solution presents technical limitations as well as propensity for failure in case of accidents, explosions, fire, flooding, and burial [1].

As in most far-field wireless communication systems, the previous solution uses radio-frequencies (RF) with radiating antennas and a transmission medium with characteristics similar to the vacuum. An alternative is to employ a transmission technique that propagates through the soil and rocks, which reduces the need for a fixed infrastructure. Such systems, that employ low-frequency electromagnetic waves for establishing a link between the surface and the underground mine, are called through-the-earth (TTE) communication systems, and are depicted in Figure 1.

In underground propagation, however, the presence of rocks, soil, water, minerals, and other materials with non-negligible electric conductivity, results in a very deep attenuation of higher frequencies. Consequently, we have to employ lower frequencies only, which have a better propagation through the earth, but in turn severely limit the bandwidth available for information transmission [2]. Transmission is usually done by magnetic induction, with frequencies below $30 \mathrm{kHz}$. Besides the narrow bandwidth and large propagation loss, TTE systems are also susceptible to atmospheric noise and alternate-current harmonics from transmission lines and electrical equipment, which also represent impairments to communication performance, especially in the uplink [2].

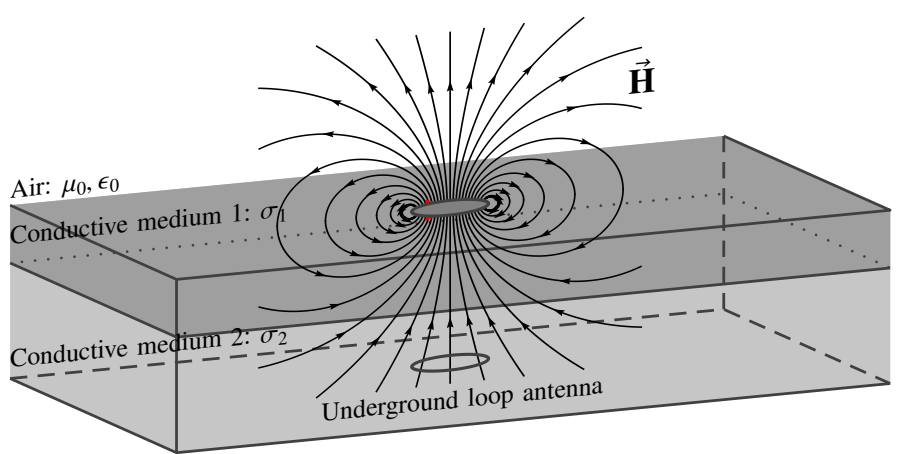

Fig. 1: Schematic representation of a TTE communication system in the downlink.

In particular, communication to and between people working in confined spaces has always been a vital matter to the mining industry, and, especially in emergency situations, it is desirable to keep uninterrupted and live contact between the rescue team and trapped personnel. A deliberation from the US congress demanding an emergency communication system 
for underground coal mines during accidents, named Mine Improvement and New Emergency Response (MINER) Act, promoted research and development of TTE technology, as it is the only remaining communications technique employable in these situations. According to this law, such systems must be wireless and bidirectional, as well as provide communication from surface to underground tunnels and be capable of locating people locked inside mines [3]. This was the initial motivation for the industry to develop commercial TTE solutions, but, as mentioned at the beginning of this introduction, other potential applications have recently emerged.

TTE transmission is clearly a technology with many potential applications, but some physical and technical challenges still limit its widespread commercial usage. Among those, one can list: antenna physical sizes, power levels associated with intrinsically safe transmission and small available bandwidth. In this contribution we discuss these technical challenges and transmission techniques that can be employed in TTE systems.

Firstly, in Section II we briefly discuss the alternatives for communications in underground mines, including, but not restricted to TTE. In Section III we present the propagation channel model of TTE communications system, and in Section IV we present the noise characteristics of low frequency systems, such as TTE. Then, using these models, we show some magnetic-field and link-level simulation results in Section V. In Section VI we discuss some aspects related to higher layers in TTE systems, and, finally, in Section VII we make some concluding remarks and discuss future work on TTE.

\section{Underground Mine COMmunications}

A mine can be defined as an individual mass of mineral or fossil substance in excavation, and, whether it is on the surface or underground, it must have economical value [4]. Underground mines are usually extremely humid, with relative humidity levels around $95 \%$ or more. Corrosive water, dust, explosive and toxic gases, such as carbon dioxide and methane, are substances that can affect the performance of personnel and equipment, including communication devices. This scenario is similar to the one found in tunnels and subways, but with more adverse conditions.

In underground mine environments, it is possible to use either wired communication techniques, called through-thewire (TTW), or wireless techniques. These latter can be either through-the-air (TTA), using radio waves, or through-the-earth (TTE).

Regarding the electric characteristics of the medium, mines differ from each other not only by their compounding minerals, but also by the proportion of this material in relation to the others in the overburden separating the surface from the minerals of interest. The electric conductivity of the medium sharply increases the channel losses. Conductivity can also define the kind of equipment and configuration chosen for the selected communication system. For example, the electric conductivity of walls can influence reflection and diffraction coefficients, as well as the channel temporal dispersion.

Before a detailed analysis of TTE, both TTW and TTA are discussed briefly on the following subsections, so that we can understand the advantages and challenges of each alternative.

\section{A. Through-The-Wire (TTW) Communications}

TTW systems use guided media [5] both for communication inside the mine and for underground-to-surface communication. One of the first instruments for mine communication was the magnetic telephone [6], which was based on wired lines for voice links. A magnetic generator was manually started using a lever, producing enough alternate current to activate the bells from other connected telephones. Once the connection was established, batteries were used to keep the system powered.

Currently, fiber optics are increasingly being used, as they permit high transmission rates through long distances inside a mine, reaching distances of nearly $70 \mathrm{~km}$ without signal regeneration [7]. Because of their great capacity, they are used for real-time monitoring of several activities, such as fire protection or automatic systems. However, deployment of fiber optics is costly and inflexible.

Alternatively, leaky feeders can be employed. Instead of using an external metallic shielding for electromagnetic interference protection, these coaxial cables use a thin copper layer with small perforations, that operate as an aperture antenna array. In consonance with its irradiation and receiving characteristics, these structures can be seen as a hybrid between TTW and TTA. Due to the power attenuation of cables, it is necessary to use amplifiers located at regular distances, typically from 350 to $500 \mathrm{~m}$. Leaky feeders support bidirectional communication, commonly for VHF and UHF [8].

\section{B. Through-The-Air (TTA) Communications}

One particular characteristic of all mines, including underground ones, is continuous transformation, as a result of mineral and waste rock extraction, making deployment of wired techniques challenging. For communication inside a mine, wireless TTA systems are, therefore, usually more adequate than wired systems, due to their easy installation and adaptation to the mine expansion. However, even for TTA systems, coverage expansion normally requires an increase of the telecommunications infrastructure [5].

The mining environment is considerably different from the one found in typical wireless terrestrial systems. Hence, when planning a TTA system, one must consider some particular mine attributes, that have a large influence on the system performance, such as mine shape and type of access. For example, underground tunnels have a tendency to act as waveguides leading to low propagation losses along galleries.

Many TTA systems are built upon standard wireless communication systems, particularly wireless local area networks (WLAN), that specify lower layers protocol, such as IEEE 802.15.4 or some modification of IEEE 802.11 [9].

Considering node distribution and infrastructure limitation in mines, several papers indicate the use of ad hoc networks [10], [11], [12], [13], [14]. In these, a crucial factor is protocol performance, especially those for access and routing control.

Finally, the applicability of 5G technologies in underground mines is currently under investigation[15].

\section{Through-The-Earth (TTE) Communications}

In the years following the MINER Act, NIOSH (National Institute for Occupational Safety) promoted the development 
of a series of communication and location technologies for underground mines [3]. This has led to prototypes developed by five different companies: Alertek, E-Spectrum Tecnologies, Lockheed Martin, Stolar e Ultra Electronics. Four prototypes were based on magnetic field detection using loop antennas and the other one was based on electric field detection.

The proposed TTE systems have shown the capability of establishing unidirectional communication and, in some cases, bidirectional communication for voice and text up to $300 \mathrm{~m}$ (voice) and $600 \mathrm{~m}$ (text) depth. For voice transmission, those prototypes used frequencies from $3150 \mathrm{~Hz}$ to $4820 \mathrm{~Hz}$. Some prototypes also possess a localization mode where only a tone is transmitted in the uplink. By triangulating the signals received at surface it is possible to locate the transmitter and hence trapped miners.

On some prototypes analogical SSB (Single-side band) modulation was used, and on others digital modulation PSK (Phase shift keying) and/or FSK (Frequency shift keying). In some prototypes noise cancelling techniques [3] were also tested.

Additionally, a few projects listed below became commercial products [16]:

- Flex Alert, manufactured by the Canadian company MineRadio Systems is a unidirectional communication system providing a link from the surface to underground galleries, for personnel evacuation in case of emergency. It uses magnetic field at low frequencies transmitting information to miners' helmets. It consists of a loop antenna with 10 to $120 \mathrm{~m}$ length, strategically positioned over the mine. In emergency situations, a signal is emitted from surface to all miners, making their helmet lamps flash [7].

- Personal Emergency Device (PED), from the Australian company MineSite Technology, is a unidirectional communication system providing SMS transmission to personnel inside the mine wirelessly. Even though it allows downlink communications only, it can be complemented by leaky feeders to provide the uplink [7]. This system is also used for remote activation of explosives and equipment control.

- After joint tests with NIOSH, Lockheed Martin started commercializing the MagneticLink MCS, which is a selfsufficient bidirectional TTE communication system that supports voice, text and localization, based on lowfrequency magnetic waves. Tests at $500 \mathrm{~m}$ depth validated voice and text applications, using a $130 \mathrm{~m}$ length antenna on the mine surface and a multiple-loop antenna inside the mine.

- The Canadian company Vital Alert [17] recently developed a digital TTE system named Canary, providing bidirectional data and voice transmission. Canary's receiver is implemented by means of software-based radio, making it reconfigurable for an operation frequency from $300 \mathrm{~Hz}$ to $9 \mathrm{kHz}$. This device permits adaptive modulation with data rate from 9 bps to 1 kpbs.

Regarding the modulation and signal processing, an overview can be found in [2], which suggests the use of MSK (minimum shift keying) as a modulation technique.
This reference also proposes techniques for atmospheric noise cancelling and error correcting codes in order to provide more robustness to the system. Also, it affirms that it is possible to enhance signal-to-noise ratio (SNR) from 10 to $30 \mathrm{~dB}$ using multiple orthogonal antennas and by the application of other techniques, such as adaptive noise cancellation, maximum likelihood detection and decision feedback. Bearing in mind the significant advances in wireless communications in the past couple of decades, the lack of recent studies related to signal processing for TTE communication can indicate potential gains to be obtained in this area.

\section{TTE PROPAGATION ENVIRONMENT}

Due to intrinsic soil characteristics, such as the electric conductivity $\sigma$, the permittivity $\epsilon$ and the permeability $\mu$, the penetration of the magnetic field $H$ in a conductive medium obeys the diffusion equation $\nabla^{2} H=\mu \sigma \partial H / \partial t$, instead of the wave equation $\nabla^{2} H=\mu \varepsilon\left(\partial^{2} H\right) /\left(\partial t^{2}\right)$, with an exponentially decaying field as a function of distance, angular frequency $\omega$ and electric conductivity of the proapagation medium. In a good conductor (with $\sigma(\epsilon \omega)>>1$ ), the signal attenuation is described by the skin depth $\delta=\sqrt{2 /(\omega \mu \sigma)}$, which is a reference distance, at which a plane wave decays to $1 / e$ (or -8.7 $\mathrm{dB}$ ) of its strength, when propagating in a conductive medium. That inverse square root dependency on the frequency justifies the use of low frequencies, usually below $30 \mathrm{kHz}$ for TTE communication.

Propagation in a conductive medium also alters some basic wave characteristics, such as propagation velocity and wavelength. In a good conductor, the real part of the complex permittivity may be neglected and the wavelength of transmitting signal can be described by $\lambda_{\text {soil }}=2 \pi \delta$. For instance, in a TTE system operating at $10 \mathrm{kHz}$ in a soil with $\sigma=10^{-3} \mathrm{~S} / \mathrm{m}$, the wavelength can decrease 30 times when compared with open space conditions. Therefore, approximations for electromagnetic field zones commonly used in the literature may be different when applied to propagation through soil.

\section{A. Field Zones}

Field zones are useful to distinguish electrical distances at which electric or magnetic fields behave differently. That behavior may be the degree of dependence of the field on its source, or if the time-variant field behaves as a static field, or if the field behaves as a plane wave, etc. Traditionally, field zones for transmission in vacuum or free space are divided in: reactive near field, radiating near field, transition zone and far field. In both near-field zones, the electromagnetic field is a consequence of wave interference originated in several points of the antenna structure. In the far field, magnetic and electric field are in phase and have a fixed relation between each other, the transmission antenna is seen as a point source, and its field can be seen as a plane wavefront. In the transition zone, both behaviours may be observed. In a conductive medium, Gibson [18] proposed a different subdivision for those field zones.

In regions very close to the transmission antenna, even when there are time variations, the electromagnetic field presents a quasi-static behaviour following static laws, with attenuation 
following an inverse cube law of the distance. Beyond the near field, the conductive medium starts to contribute with field attenuation. In the far-field zone, even though there are medium losses, the electromagnetic field obeys the law of attenuation with the inverse of the distance. Here, the propagation mechanism is determined by Foucault currents induction on a conductive media. Finally, the transition zone is an arbitrary zone between the near field and far field.

Table I presents the field zones and their conditions for the vacuum and for a conductive medium. A system operating in free space at $10 \mathrm{kHz}\left(\lambda_{0}=30 \mathrm{~km}\right)$ with antenna separation of $300 \mathrm{~m}$ is the in reactive near field $\left(\frac{\lambda_{0}}{2 \pi} \approx 4775 \mathrm{~m}\right)$, whereas, in a conductive medium with $\sigma=10^{-3} \mathrm{~S} / \mathrm{m}$ and $\mu=\mu_{0}$, we have $\lambda_{\text {soil }} / 2 \pi=\delta \approx 160 \mathrm{~m}<300 \mathrm{~m}$, i.e., it is in the transition zone and closer to the far field than to the near field.

\begin{tabular}{lll} 
Model & Type of approximation & Conditions \\
\hline \multirow{3}{*}{ Vacuum } & Reactive near field & $r<\lambda_{0} / 2 \pi$ \\
& Radiating near field & $\lambda_{0} / 2 \pi<r<\lambda_{0}$ \\
& Transition zone & $\lambda_{0}<r<2 \lambda_{0}$ \\
& Far field & $r>2 \lambda_{0}$ ou $2 D^{2} / \lambda_{0}$ \\
\hline \multirow{3}{*}{ Conductive } & Quasi-static & $r<<\lambda_{\text {soil }} / 2 \pi$ \\
medium & Near field & $r^{2}<<\left(\lambda_{\text {soil }} / 2 \pi\right)^{2}$ \\
& Transition Zone & $r \approx \lambda_{\text {soil }} / 2 \pi$ \\
& Far field & $r>>\lambda_{\text {soil }} / 2 \pi$ \\
\hline
\end{tabular}

TABLE I: Vacuum and conductive medium field zones

\section{B. Magnetic Fields Models}

The magnetic moment $m_{d}=N_{t x} I_{t x} S_{t x}$ of a loop antenna indicates the inductive transmission capacity as a function of the transmitter characteristics. $N_{t x}$ is the number of windings at the transmitters, $I_{t x}$ is the RMS value of a tonal electric current at the transmission antenna, and $S_{t x}$ denotes the loop area. According to this formula, it is possible to note that an increase in the magnetic moment also increases the power dissipated as heat, $P_{t x}=R_{t x} I_{t x}^{2}$, where the loop resistance $R_{t x}$ increases with $N_{t x}$ and/or $S_{t x}$. Even though this power is dissipated and not irradiated, it determines the current value used in the magnetic field generation and, because of that, it is associated indirectly with the transmission power.

The simplest approximation for a magnetic field created by an electrically small loop antenna is made by considering vacuum as a homogeneous medium, neglecting any boundary condition [19] and assuming a uniformly distributed current on the loop. At a very close distance $r$ to the source $(r<<\lambda / 2 \pi)$, the intensity of a time varying magnetic field is similar to a static field calculated by Biot-Savart law [20]. In the vacuum at $10 \mathrm{kHz}$, this distance is about hundreds of meters. For a loop antenna, the quasi-static magnetic field can be described by:

$$
\boldsymbol{H}_{q e}=\frac{m_{d}}{4 \pi r^{3}}\{2 \cos (\theta) \hat{\boldsymbol{r}}+\sin (\theta) \hat{\boldsymbol{\theta}}\},
$$

where $\hat{\boldsymbol{r}}, \hat{\boldsymbol{\theta}}$ are vectors in radial and elevation spherical coordinates. The model adaptation from an infinite plane in the vacuum [19] to a conductive infinite medium (CIM) is realized by altering only the wave number $k_{\text {soil }}=(1-j) / \delta$. This field approximation for both uplink and downlink, in spherical coordinates, is described in its phasorial mode by [18]:

$$
\begin{aligned}
\boldsymbol{H} & =\frac{m_{d}}{4 \pi r^{3}} e^{-j T} e^{-T}\{2 \cos \theta(1+(1+j) T) \hat{\boldsymbol{r}}+ \\
& \left.+\sin \theta\left(1+(1+j) T+2 j T^{2}\right) \hat{\boldsymbol{\theta}}\right\},
\end{aligned}
$$

where $T=r / \delta=r \sqrt{\mu \sigma \omega / 2}$ denotes the transmission range, normalized by skin-depth, and contemplates losses due to the soil. Parameter $T$ is dimensionless and can also be interpreted as a spacial frequency normalized by a known transmission range $r$.

Wait [21], [22] formulated analytic expressions for the magnetic field generated by circular antennas with a uniform current, separating both surface and underground transmission media, with a so called homogeneous semi-space model (HSS). Fields for uplink $\boldsymbol{H}_{u p}$ [21] and downlink $\boldsymbol{H}_{\text {down }}$ [22] in cylindrical coordinates in both radial $\hat{\rho}$ (horizontal field) and depth $\hat{z}$ (vertical field) directions are respectively given by:

$$
\begin{gathered}
\boldsymbol{H}_{u p}=\frac{m_{d}}{2 \pi h^{3}} \int_{0}^{\infty} \beta x\left\{J_{0}(D x) \hat{z}-J_{1}(D x) \hat{\boldsymbol{\rho}}\right\} d x, \\
\boldsymbol{H}_{\text {down }}=\frac{m_{d}}{2 \pi h^{3}} \int_{0}^{\infty} \beta(x)\left\{J_{0}(D x) \hat{z}-\right. \\
\left.-J_{1}(D x) \frac{\left(x^{2}+j 2 \mathcal{T}^{2}\right)^{1 / 2}}{x} \hat{\boldsymbol{\rho}}\right\} d x,
\end{gathered}
$$

where

$$
\beta(x)=\frac{J_{1}(A x)}{A x / 2} \frac{x^{3}}{\left(x^{2}+j 2 \mathcal{T}^{2}\right)^{1 / 2}+x+\chi} e^{-Z x} e^{-\left(x^{2}+j 2 \mathcal{T}^{2}\right)^{1 / 2}},
$$

and $A=a / h, D=\rho / h, Z=h_{0} / h, \mathcal{T}=h / \delta . a$ is the loop radius, $h_{0}$ and $h$ are, respectively, the distances between the surface antenna and the soil, and between the soil and the underground buried antenna; $J_{0}$ and $J_{1}$ are Bessel functions of the first kind, and $\chi$ is zero for Wait's approximations, but it will be used just ahead in Durkin's model. Figure 2 illustrates the geometry for fields in this HSS scenario.

We observe that there is a reciprocity between the two links for the vertical field, but not for the horizontal field, that, in fact, could be ignored in a configuration in which the antennas are coaxially aligned, i.e., when $\rho=0$ (or $\mathcal{T}=T$ ) making $J_{1}(D x)=0$. Likewise, in the CIM model in (2), for $\theta=$ $180^{\circ}$ in coaxial configuration, the elevation field component disappears, and only the vertical field component remains at the $\hat{\boldsymbol{r}}$ direction. In contrast with the CIM model, in which the loop must have sufficiently small dimensions to ensure that the current has spatial uniformity, the HHS models have the correction factor $J_{1}(A x)$, which compensates for the antenna size variation.

Durkin [23] suggests that at the interface between earth and air there exists a barrier, that can be modeled by a thin layer of conductivity greater than the conductivity of the homogeneous half-space below it. His model includes the term $\chi=j 2 \mathcal{T}^{2} \frac{h_{1}}{h} \sigma_{1}$ in the denominators of (3) and (4), where $h_{1}$ and $\sigma_{1}$ denote the height and the conductivity of the thin 


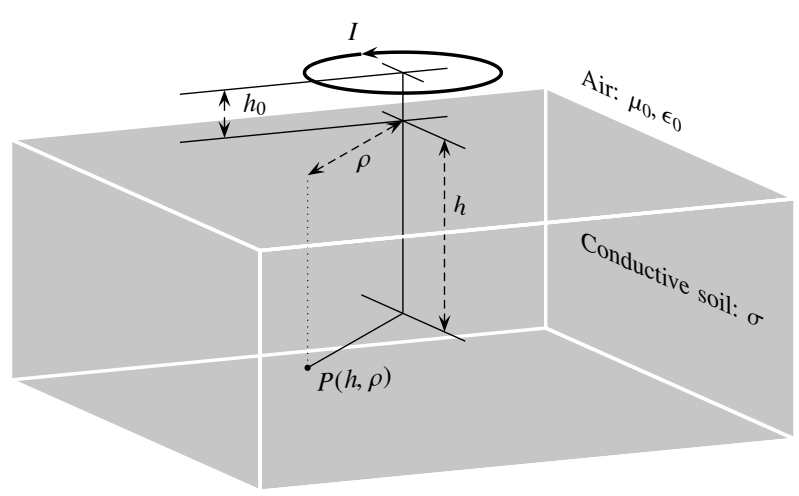

Fig. 2: Geometry used for the calculation of the magnetic field at point $P(h, \rho)$ for a circular loop antenna $h_{0}$ above the ground.

interface layer.

All the above models are on the frequency domain, since $\delta$ varies with $\omega$. It is worth noting that any linear and nonlinear transmitter distortions are excluded from the models. Nearfield and far-field approximations for these equations can be made if the conditions in Table I hold, remembering that $\delta=$ $r / T=\lambda_{\text {soil }} / 2 \pi$.

Figure 3 shows the magnetic field strength of a loop antenna along its axis $(\theta=0)$, normalized by the intensity of the quasi-static field as a function of $T$ for the CIM and HSS models, and we can see that in both models the results are approximately the same. It also presents the field strength at a point on the same loop plane $(\theta=\pi / 2)$, only for the CIM model, as the HSS model is not adapted for coplanar configuration. In this figure we observe the excess loss (or gain) due to the medium and the structure of the loop antenna beyond the cubic inverse law of distance.

For a more precise appreciation of the field strength variation with distance, we could add an attenuation of $60 \mathrm{~dB}$ per decade for a fixed frequency. We observe that up to the distance $T=1 / 4$, which can amount to $40 \mathrm{~m}$ in a system operating at $1 \mathrm{kHz}$ with moderate conductivity $\sigma=10^{-2} \mathrm{~S} / \mathrm{m}$, the models follow virtually the behaviour of the quasi-static field. From there on, the coplanar field displays gain until $T \approx 3$, when it starts to present attenuation, while the coaxial field always has its intensity reduced, initially by $1.5 \mathrm{~dB}$ after the first $\delta(T=1)$, then by $4.5 \mathrm{~dB}$ between the second and first $\delta$, and finally converging to $8.7 \mathrm{~dB} / \delta$ at $T \rightarrow \infty$, like the coaxial field. This convergence value of the attenuation rate $/ \delta$ is typical for plane waves at the far field and it is used to define $\delta$ itself $\left(20 \log _{10}\left(e^{-\frac{r}{\delta}=-1}\right)=-8.7 \mathrm{~dB}\right)$.

For a fixed distance, the spectrum as a function of the normalized frequency $T$ (or $\mathcal{T}$ ) shows a low-pass filter behaviour, with $3 \mathrm{~dB}$ bandwidth equal to 1.4 for the coaxial field, and 2.8 for the coplanar field. However, we will see in Subsection III-C that these are not the channel frequency response curves between the two communicating nodes.

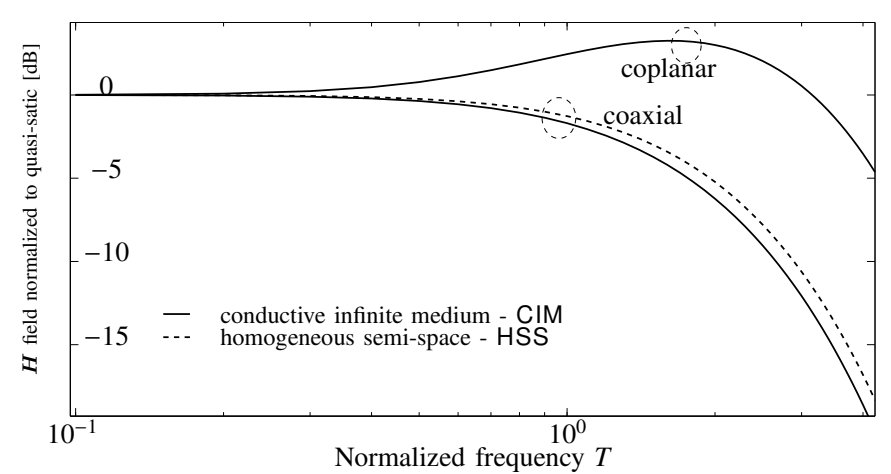

Fig. 3: Magnetic field strenght of a loop antenna for coaxial and coplanar configuration for CIM and HSS models (uplink).

\section{Channel Modelling and Optimal Operation Frequency}

In systems that operate in moderately low frequencies, it is more common to use voltage and current to to parametrize quadripoles and, consequently, parameters based on impedances are used. To analyse the propagation channel at frequencies below $100 \mathrm{kHz}$, it is useful to adopt the concept of transfer impedance between loops, that considers the phasor current $I_{t x}(\omega)$ in the transmission loop and the induced voltage $V_{r x}(\omega)$ at the receiving antenna:

$$
Z(\omega)=V_{r x}(\omega) / I_{t x}(\omega) .
$$

According to Faraday's law, the voltage induced by a magnetic field that goes through a conductive closed loop depends on the temporal variation of the magnetic flux that enters the loop orthogonally [20]. As a result, the voltage induced at the antenna due to the magnetic field is given by:

$$
\begin{aligned}
V_{r x}(\omega) & =-j \omega N_{r x} \int_{S} \mu \boldsymbol{H} \cdot \boldsymbol{d} \boldsymbol{S}= \\
& =-j \omega \mu N_{r x} S_{r x} H \cos (\varphi),
\end{aligned}
$$

where $N_{r x}$ e $S_{r x}$ are the number of turns and the area of the receiving loop, respectively, and $\varphi$ is the angle between the magnetic field and the loop axis, that is orthogonal to its plane. We see in (7) that, although the field attenuates at high frequencies, the receiver causes the output signal to also be attenuated at low frequencies. In spherical coordinates, if $\varphi=\theta$, both loops planes are parallel to each other. Expressing $\boldsymbol{H}$ in spherical coordinates, $\boldsymbol{H}_{\boldsymbol{r}}$ at the $\hat{\boldsymbol{r}}$ direction, and $\boldsymbol{H}_{\boldsymbol{\theta}}$ at the $\hat{\boldsymbol{\theta}}$ direction, we have,

$$
\begin{aligned}
V_{r x}(\omega)=-j \omega \mu N_{r x} S_{r x} & {\left[\boldsymbol{H}_{\boldsymbol{r}} \cos \left(\theta-\theta_{x}\right) \cos \left(\theta_{y}\right)-\right.} \\
& \left.-\boldsymbol{H}_{\boldsymbol{\theta}} \sin \left(\theta-\theta_{x}\right) \cos \left(\theta_{y}\right)\right],
\end{aligned}
$$

where $\theta_{x}$ e $\theta_{y}$ are rotation angles for the $\hat{\mathbf{x}}^{\prime}$ and $\hat{\mathbf{y}}^{\prime}$ axis, that are auxiliary axis created by the $\phi-90^{\circ}$ rotation of the $\hat{\mathbf{z}}$ axis, where $\phi$ is the azimuthal angle. Figure 4 illustrates the described geometry. Using (2) and (8), we have: 


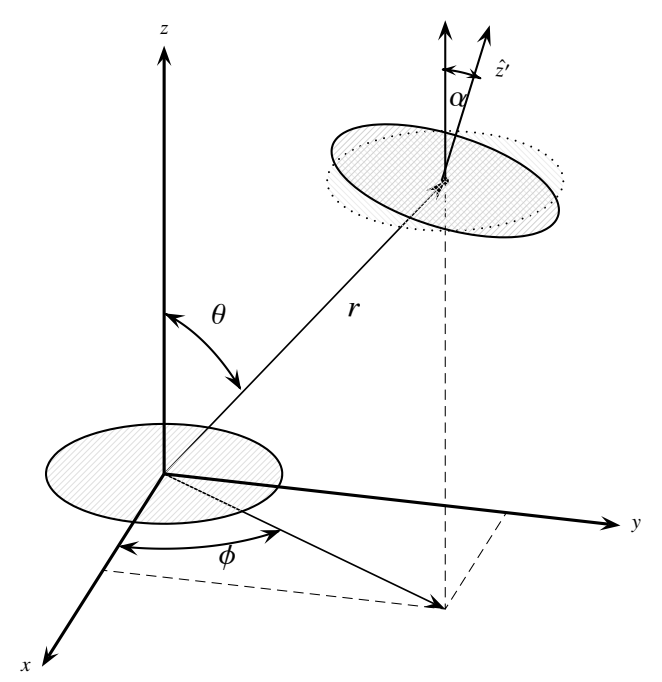

Fig. 4: Geometry used for the CIM model in spherical coordinates. The tilt angle $\alpha$ formed by orthogonal vectors to the planes determined by the two antennas is the result of rotations around the $\hat{\mathbf{x}}^{\prime}$ e $\hat{\mathbf{y}}^{\prime}$ axis.

$$
\begin{aligned}
V_{r x}(\omega)= & -j \omega \mu N_{r x} S_{r x} \frac{N_{t x} S_{t x} I_{t x}(\omega)}{4 \pi r^{3}} e^{-j T} e^{-T} \\
& {\left[2(1+(1+j) T) \cos (\theta) \cos \left(\theta-\theta_{x}\right) \cos \left(\theta_{y}\right)-\right.} \\
& \left.\left(1+(1+j) T+2 j T^{2}\right) \sin (\theta) \sin \left(\theta-\theta_{x}\right) \cos \left(\theta_{y}\right)\right] .
\end{aligned}
$$

Replacing $\frac{\mu \omega}{2}=\frac{T^{2}}{r^{2} \sigma}$ and rearranging (9), we get:

$$
\begin{aligned}
& V_{r x}(\omega)=N_{t x} N_{r x} S_{t x} S_{r x} I_{t x} {\left[\sqrt{G_{r}^{t x} G_{r}^{r x}} \boldsymbol{F}_{r}+\right.} \\
&\left.+\sqrt{G_{\theta}^{t x} G_{\theta}^{r x}} \boldsymbol{F}_{\boldsymbol{\theta}}\right],
\end{aligned}
$$

where

$$
\begin{gathered}
\boldsymbol{F}_{\boldsymbol{r}}=\frac{T^{2} \sqrt{1+2 T+2 T^{2}}}{\pi \sigma r^{5}} e^{-T} e^{j\left\{\tan ^{-1}\left(\frac{T}{1+T}\right)-T-\frac{\pi}{2}\right\}}, \\
\boldsymbol{F}_{\boldsymbol{\theta}}=\frac{T^{2} \sqrt{(1+T)^{2}+\left(T+2 T^{2}\right)^{2}}}{2 \pi \sigma r^{5}} e^{-T} e^{j\left\{\tan ^{-1}\left(\frac{T+2 T^{2}}{1+T}\right)-T+\frac{\pi}{2}\right\}},
\end{gathered}
$$

and the normalized gain of the transmission and reception antennas, polarized at the directions $\hat{\boldsymbol{r}}$ and $\hat{\boldsymbol{\theta}}$ are $G_{r}^{t x}=$ $\cos ^{2}(\theta), G_{r}^{r x}=\cos ^{2}\left(\theta-\theta_{x}\right) \cos ^{2}\left(\theta_{y}\right), G_{\theta}^{t x}=\sin ^{2}(\theta)$ and $G_{\theta}^{r x}=\sin ^{2}\left(\theta-\theta_{x}\right) \sin ^{2}\left(\theta_{y}\right)$.

Then, adopting the concept of transfer impedance between loops, that considers the phasor current $I_{t x}(\omega)$ in the transmission loop and the induced voltage $V_{r x}(\omega)$ at the receiving antenna, and substituting (10) in (6) we get:

$$
\begin{aligned}
& Z_{\mathrm{CIM}}(T)=N_{t x} N_{r x} S_{t x} S_{r x} {\left[\sqrt{G_{r}^{t x} G_{r}^{r x}} \boldsymbol{F}_{\boldsymbol{r}}+\right.} \\
&\left.+\sqrt{G_{\theta}^{t x} G_{\theta}^{r x}} \boldsymbol{F}_{\boldsymbol{\theta}}\right] .
\end{aligned}
$$

In $Z_{\mathrm{CIM}}(T)$, all linear distortions are in $\boldsymbol{F}_{\boldsymbol{r}}$ and $\boldsymbol{F}_{\boldsymbol{\theta}}$, including the derivative operation performed by the receiving loop antenna. This facilitates the separation of contributions throughout the network as a function of the type of linear system.

For the HSS model in cylindrical coordinates, the voltage induced as a function of the fields $\boldsymbol{H}_{\boldsymbol{z}}$ in the $\hat{\boldsymbol{z}}$ direction, and $\boldsymbol{H}_{\boldsymbol{\rho}}$, in the $\hat{\boldsymbol{\rho}}$ direction, is given by:

$$
\begin{aligned}
V_{r x}(\omega)=-j \omega \mu N_{r x} S_{r x} & {\left[\boldsymbol{H}_{\boldsymbol{z}} \cos \left(\theta_{x}\right) \cos \left(\theta_{y}\right)+\right.} \\
& \left.+\boldsymbol{H}_{\boldsymbol{\rho}} \sin \left(\theta_{x}\right) \cos \left(\theta_{y}\right)\right] .
\end{aligned}
$$

Then, the transfer impedance $Z_{\mathrm{HSS}}(\mathcal{T})$ and the transfer functions $\boldsymbol{F}_{\boldsymbol{z}}, \boldsymbol{F}_{\boldsymbol{\rho}}^{\boldsymbol{u p}}$ and $\boldsymbol{F}_{\boldsymbol{\rho}}^{\boldsymbol{d o w n}}$ may be derived in similar way as for the CIM model and given by:

$$
\begin{gathered}
Z_{\mathrm{HSS}}(\mathcal{T})=N_{t x} N_{r x} S_{t x} S_{r x}\left[\sqrt{G_{z}^{r x}} \boldsymbol{F}_{\boldsymbol{z}}+\sqrt{G_{\rho}^{r x}} \boldsymbol{F}_{\boldsymbol{\rho}}\right] \\
\boldsymbol{F}_{\boldsymbol{z}}=\frac{\mathcal{T}^{2}}{\pi \sigma r^{5}} e^{-j \frac{\pi}{2}} \int_{0}^{\infty} \beta(x) J_{0}(D x) d x \\
\boldsymbol{F}_{\boldsymbol{\rho}}^{\boldsymbol{u p}}=\frac{\mathcal{T}^{2}}{\pi \sigma r^{5}} e^{-j \frac{\pi}{2}} \int_{0}^{\infty} \beta(x) J_{1}(D x) d x, \text { and } \\
\boldsymbol{F}_{\boldsymbol{\rho}}^{\boldsymbol{d o w} \boldsymbol{n}}=\frac{\mathcal{T}^{2}}{\pi \sigma r^{5}} e^{-j \frac{\pi}{2}} \int_{0}^{\infty} \beta(x) J_{1}(D x) \frac{\left(x^{2}+j 2 T^{2}\right)^{1 / 2}}{x} d x,
\end{gathered}
$$

where $G_{z}^{r x}=\cos ^{2}\left(\theta_{x}\right) \cos ^{2}\left(\theta_{y}\right)$ and $G_{\rho}^{r x}=\sin ^{2}\left(\theta_{x}\right) \cos ^{2}\left(\theta_{y}\right)$. In this model, part of the antennas normalized gains are accounted for inside the channel transfer function and depend mainly on $\rho, h, \theta_{x}$ and $\theta_{y}$.

The calculation of the power delivered to the receiver as a function of system parameters is important for a number of performance measurements in communications systems.

The relationship between the dissipated powers in the first loop at the transmitter and the delivered power to the second loop at the receiver is given by:

$$
\frac{P_{r x}}{P_{t x}}=\frac{|Z(T)|^{2}}{R_{t x} R_{r x}},
$$

from which we get

$$
\begin{aligned}
& \sqrt{P_{r x, \mathrm{CIM}}}=\sqrt{P_{t x}} \Phi_{t x} \Phi_{r x} \mid \sqrt{G_{r}^{t x} G_{r}^{r x}} \boldsymbol{F}_{r}+ \\
& +\sqrt{G_{\theta}^{t x} G_{\theta}^{r x}} \boldsymbol{F}_{\boldsymbol{\theta}} \\
& \sqrt{P_{r x, \mathrm{HSS}}}=\sqrt{P_{t x}} \Phi_{t x} \Phi_{r x}\left|\sqrt{G_{z}^{r x}} \boldsymbol{F}_{z}+\sqrt{G_{\rho}^{r x}} \boldsymbol{F}_{\boldsymbol{\rho}}\right|,
\end{aligned}
$$

where $\Phi_{t x}$ and $\Phi_{r x}$ are the specific apertures of the transmitting and receiving antennas, respectively. The specific aperture of a loop antenna is a function of its radius $a$, mass $M$, mass density $\rho_{l}$, and wire conductivity $\sigma_{l}$, and is given by $\Phi=0.5 a \sqrt{M \sigma_{l} / \rho_{l}}$.

Both $Z(T)$ and $P_{r x} / P_{t x}$ carry intrinsic parameters of the used loops. On the other hand, the transfer functions $\boldsymbol{F}$ that do not depend on these parameters and therefore will be used to analyze the behavior of the physical channel. Figure 5 presents the magnitude spectrum of the channel transfer function for antennas at the coaxial and coplanar configurations.

We observe that the channels are passband and, for the coaxial configuration geometry, the normalized optimal fre- 


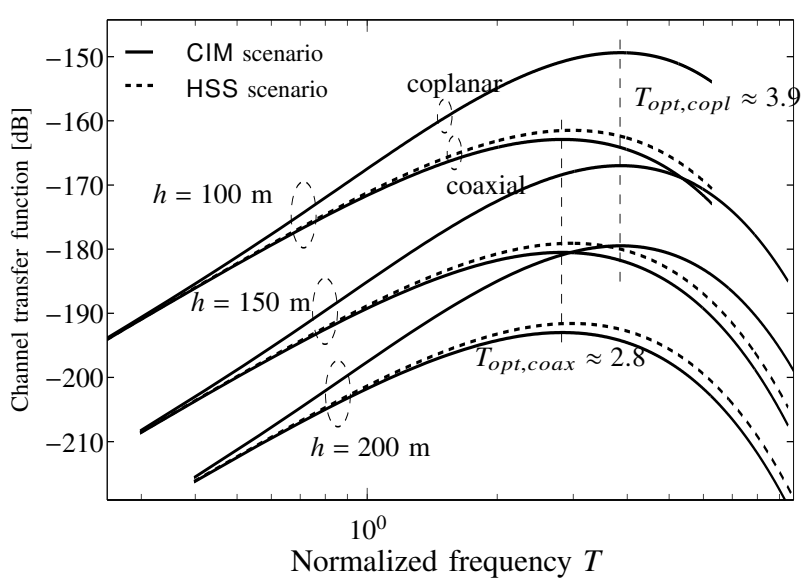

Fig. 5: Channel transfer function between two loop antennas in coaxial and coplanar configurations for the CIM and HSS models. (uplink)

quency, i.e., the one with the highest gain, is found to be $T_{\text {opt }} \approx 2.83$. Interpreting the channel as a filter, it is possible to find its quality factor as $Q=2.2$ and to notice that the shape of channel frequency response is asymmetrical relative to $T_{o p t}$. For example, considering a depth of $r=200 \mathrm{~m}$ and soil conductivity $\sigma=10^{-2} \mathrm{~S} / \mathrm{m}$, the optimal frequency $f_{\text {opt }} \approx 5$ $\mathrm{kHz}$ and the bandwidth in which there is less than $3 \mathrm{~dB}$ variation is $11 \mathrm{kHz}$. For coplanar transmission, $T_{\text {opt }} \approx 3.86$ with quality factor $Q=1.8$.

In practice, no model can accurately predict the channel attenuation for any frequency and depth because the soil is rarely homogeneous. The adequacy of CIM and HSS models depends on how one is using them. When knowing the equivalent conductivity, which is an artificial value valid for a specific frequency and depth, these models may be useful to set initial transmitting parameters. When no information about soil conductivity is available, sounding tests in frequency domain may be necessary to estimate the optimal frequency for a specific depth.

\section{Antenna Modelling for TTE Communications}

The type and size of antennas for TTE communications depend on the desired transmission range and on the available physical space on site. As discussed earlier, antennas based on magnetic field, such as loops, are preferred over antennas based on electric field, such as electric dipoles or monopoles, because electric fields have higher attenuation rates in the conductive medium [18].

The magnetic moment produced by an antenna depends on the material that constitutes the antenna and on the antenna geometry. In systems that use loop antennas and that need to have a moderate range $(>300 \mathrm{~m})$, it may be necessary to employ transmission antennas with length of a few tenths of meters. Small loops with one or more windings are often used in short range communications in mines and caves.

Another type of antenna used as a receiver in TTE communications is the ferrite rod [18], constituted by a thin coil with many windings and a core made of a magnetic material, whose magnetic permeability is orders of magnitude larger than vacuum permeability. The higher number of windings and the core material compensate for the small cross area section of the antenna. This structure, although usually long (between $10 \mathrm{~cm}$ and $2 \mathrm{~m}$ ), can offer higher mobility to the radio equipment and is normally used in the underground, where the atmospheric noise is weaker.

In order to provide an example of how intrinsic parameters of magnetic antennas affect power consumption requirements, it is useful to consider a scenario for transmitting antennas only. A one-meter, single-winding loop antenna with $200 \mathrm{~g}$ mass and $1 \mathrm{~m}$ diameter produces a magnetic moment $m_{d}=$ $30 \mathrm{Am}^{2}$, dissipating $10 \mathrm{~W}$, whereas a small ferrite rod, with a little more than $1 \mathrm{~cm}$ radius and $20 \mathrm{~cm}$ length, produces an equivalent magnetic moment dissipating twice as much power. However, an antenna with $8.5 \mathrm{~kg}$ and $50 \mathrm{~m}$ diameter can produce up to $m_{d}=30 \mathrm{kAm}^{2}$ dissipating $100 \mathrm{~W}$. It is important to highlight the difficulty of using ferrite rods in light receivers for personal mobile communication, due to high power consumption, which does not prevent its use in heavier receivers used in mobile machinery.

Transmission in uplink is more critical than in downlink. Regarding the receiver, the atmospheric noise is clearly stronger in the surface than in deep mines. And with respect to the transmitter, space and power limitations within mines limits magnetic moment to be generated. Small loop antennas with several turns could be an alternative to large single-turn antennas for using in transmitters in confined spaces within mines. However, high reactance of multi-turn loops imposes practical limitation in terms of high voltage needed to produce enough magnetic moment to traverse long distances. In many cases, tuning the antenna through an opposed reactor for resonance at a specific frequency is the only way to provide acceptable field gain at the cost of reducing bandwidth [24], [25]. In [26], Rhodes utilizes multiple coaxial sub-loops in order to increase bandwidth using multiple resonances. Subloops are fed by a single voltage driver, electrically connected in parallel to each other and partially coupled due to a controlled distance between them (see Figure 6). The circuits are tuned in different frequencies in order to assure impedance matching for the band of interest. The distance between subloops is decreased until an acceptable degree of coupling is observed. Then, the resonating and damping components may be adjusted to achieve the desired summed frequency characteristics.

\section{TTE SyStem NoISE}

In order to cope with the strong attenuation caused by the propagation channel, TTE communications systems should operate at low frequencies, usually in the VLF and LF bands. However, two kinds of noise are present in these frequency bands, and they directly degrade the performance of TTE communication systems. In general, they are ignored in the design of conventional communications systems, and are referred as atmospheric noise and man-made noise.

The atmospheric noise is modeled as the superposition of a Gaussian background noise and impulsive spikes. The im- 


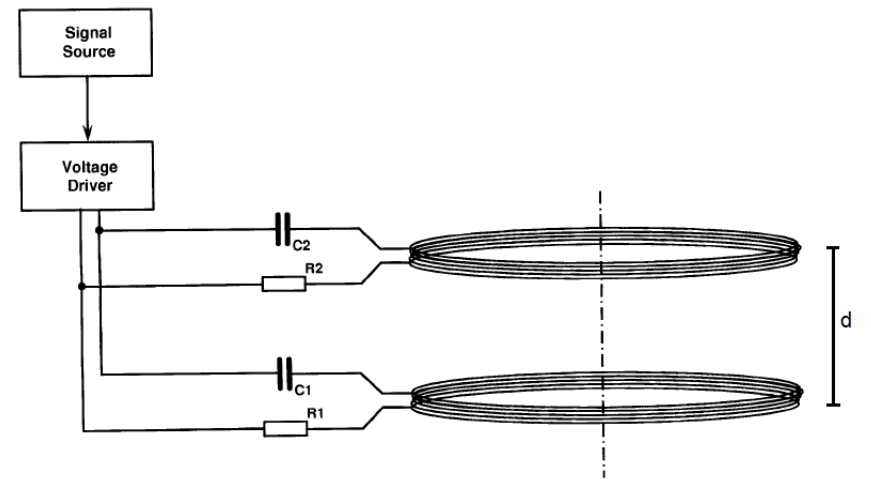

Fig. 6: Transmitting antenna composed of two partially coupled sub-loops with different resonances. Adapted from [26].

pulsive noise component is generated by lightning discharges close to the receiver (distances up to $10^{6} \mathrm{~m}$ [27]), whereas the Gaussian component is due to the aggregate of distant lightning discharges and other noise sources. The noise level varies with location, season and time of the day. The noise spectrum over a typical receiver bandwidth is essentially flat [27].

Several models for the atmospheric noise were developed, but most of them are complicated and difficult to use. The model proposed by Field and Lewinstein [28], however, is mathematically simple, easily implemented in simulations and proven to produce accurate results for a variety of noise conditions [27].

This model represents the VLF band noise as a random phasor, whose envelope is the sum of a Rayleigh random variable, that denotes the Gaussian component, and a Weibull (power-Rayleigh) random variable, that represents the impulsive component. In the time domain, the noise can be expressed by

$$
V(t)=Z(t) \cos (\omega t+\phi(t))
$$

where $Z(t)=X(t)+Y(t)$ and $\phi(t)$ are the envelope and phase, respectively. $X(t)$ is a Rayleigh random variable and $Y(t)$ is a Weibull random variable.

The Gaussian noise $X(t)$ has an envelope given by a Rayleigh random variable with probability density function (p.d.f.) given by:

$$
p_{X}(x)=\left(\frac{x}{\sigma_{0}^{2}}\right) e^{-x^{2} / 2 \sigma_{0}^{2}}, x \geq 0 .
$$

The mean and the mean power of this component are respectively given by:

$$
E[X]=(\pi / 2)^{1 / 2} \sigma_{0}=\pi^{1 / 2} R_{0} / 2,
$$

and

$$
E\left[X^{2}\right]=2 \sigma_{0}^{2}=R_{0}^{2} .
$$

The impulsive component $Y(t)$ is a Weibull random variable, whose p.d.f. is given by:

$$
p_{Y}(y)=\left(\frac{a y^{a-1}}{R^{a}}\right) e^{-y^{a} / R^{a}}, y \geq 0,
$$

where $0 \leq a \leq 2$ and $R$ denotes the average energy in $Y$ [29]. The mean and mean power of $Y(t)$ are given by:

$$
E[Y]=R \Gamma(1+1 / a)
$$

and

$$
E\left[Y^{2}\right]=R^{2} \Gamma(1+2 / a),
$$

respectively. The $a$ parameter is called spikiness [27] and strongly influences the p.d.f. shape. Also, $Г($.$) denotes the$ gamma function.

The envelope $Z(t)$ modulates a carrier, and, thus, the total noise power is given by:

$$
\begin{gathered}
P_{n}=\frac{1}{2} E\left[Z^{2}\right]=\frac{1}{2}\left\{E\left[X^{2}\right]+E[X] E[Y]+E\left[Y^{2}\right]\right\}= \\
\frac{1}{2}\left[R_{0}^{2}+\pi^{1 / 2} \Gamma\left(1+\frac{1}{a}\right) R_{0} R+\Gamma\left(1+\frac{2}{a}\right) R^{2}\right] .
\end{gathered}
$$

The impulsivity $\gamma$ gives the ratio of energy between the impulsive and the Gaussian noise components, and is given by:

$$
\gamma=\frac{E\left[Y^{2}\right]}{E\left[X^{2}\right]}=\frac{R^{2} \Gamma\left(1+\frac{2}{a}\right)}{R_{0}^{2}} .
$$

The spikiness is a function of the receiver bandwidth and characterizes the amount of impulsive noise [28]. The noise total power, the spikiness and the impulsivity are the three degrees of freedom of the Field-Lewinstein model, that can be adjusted to better characterize the noise observed in a certain environment. Usually, most of the noise power is concentrated in its impulsive component. Typical values for $\gamma$ and $a$ are: $4<\gamma<6$ and $0.25<a<0.75$ [29].

\section{A. Parameters Estimation}

In order to design optimum receivers for TTE systems, it is useful to estimate noise model parameters. One advantage of the Field-Lewinstein model is that such estimation can be performed in a relatively straightforward way, based on empirical measurements. The model has three degrees of freedom and, thus, there are three variables to be determined: $a, R_{0}$ and $R$. If the values of these variables are known, one can calculate the power of each noise component and calculate the impulsivity, $\gamma$.

To determine the three variables, we need to find a system with three equations with these variables. This is done using the moments of the observed noise [29]:

$$
E[Z]=E[X]+E[Y]=\frac{\pi^{1 / 2}}{2} R_{0}+\Gamma(1+1 / a) R,
$$

and

$$
\begin{aligned}
E\left[Z^{2}\right] & =E\left[X^{2}\right]+2 E[X Y]+E\left[Y^{2}\right]= \\
& =R_{0}^{2}+\pi^{1 / 2} \Gamma(1+1 / a) R_{0} R+\Gamma(1+2 / a) R^{2} .
\end{aligned}
$$

Similarly,

$$
\begin{aligned}
E\left[Z^{3}\right] & =E\left[X^{3}\right]+3 E\left[X^{2} Y\right]+3 E[X] E\left[Y^{2}\right]+E\left[Y^{3}\right]= \\
& =\frac{3 \pi^{1 / 2}}{4} R_{0}^{3}+3 \Gamma(1+1 / a) R_{0}^{2} R+ \\
& +\frac{3 \pi^{1 / 2}}{2} \Gamma(1+2 / a) R_{0} R^{2}+\Gamma(1+3 / a) R^{3} .
\end{aligned}
$$


Equations (31) to (33) form a nonlinear system of equations that can be solved to determine the variables $a, R_{0}$ and $R$ [29].

\section{B. Man-made Noise}

Besides the atmospheric noise, a noise component generated by human activities is observed. The harmonic components of power transmission lines usually cause the man-made noise, which is stronger at low frequencies.

Atmospheric noise is dominant between 10 and $30 \mathrm{kHz}$, while man-made noise is dominant below $1 \mathrm{kHz}$. The 50 or $60 \mathrm{~Hz}$ harmonics generated by power lines are not deterministic signals and their spectra are not located in a single frequency. They occupy a certain band centred on a harmonic, as a random signal modulates the harmonics. Also, power lines harmonics are observed even when the receiver is $1 \mathrm{~km}$ away from the nearest transmission line [27].

\section{FiELd ANd Link Simulations}

In this section, we show results of two different simulation studies. In the first case, we used a field solver for characterizing the transmission channel and in the second case we used Matlab to simulate the digital communication system in a typical TTE scenario.

\section{A. Magnetic Field Simulation}

The use of numerical methods for calculating the fields, densities and impedances from Maxwell's equations has been essential in the modelling of antenna and propagation in more complex propagation media. Commercial software, such as Computer Simulation Technology (CST) [30], used in our simulations, allows the study of electromagnetic fields on several conditions: low and high frequencies, static sources, microwave, RF, among others, using different numerical analysis methods such as FDTD (finite-difference time-domain), FEM (finite element method) or MoM (method of moments).

In the computational environment setup for the TTE transmission simulation, we used numerical FEM in the frequency domain, adjusted to the ULF/VLF bands. In a basic configuration, two loop antennas are arranged coaxially at the ends of a stratified structure composed of different materials, as shown in Figure 7. The properties of each material, such as electrical, magnetic, thermal, mechanical and density are adjusted, based on geological studies and antenna conductor types.

In order to achieve a good compromise between accuracy and processing time, the choice of suitable meshing structures is essential. We can choose between two fabric options, the tetrahedral and hexahedral. This function controls the simulation time and is available in time- or frequency-domain simulation environments. In Figure 8, we observe how this meshing changes according to the physical characteristics of the materials. For less complex structures, such as cobblestone blocks, adaptive meshing allows the use of larger cells and thus reduces the total number of nodes, reducing the number of operations.

To limit the number of numerical calculations, the boundary conditions should be chosen properly. Common options are:

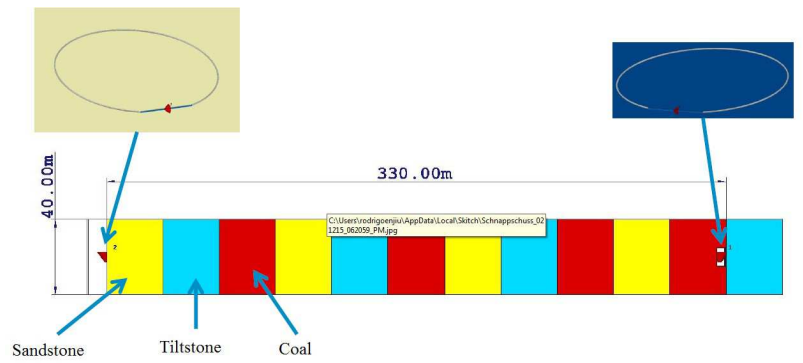

Fig. 7: Stratified model used in computational calculations of magnetic field in a TTE point-to-point link.

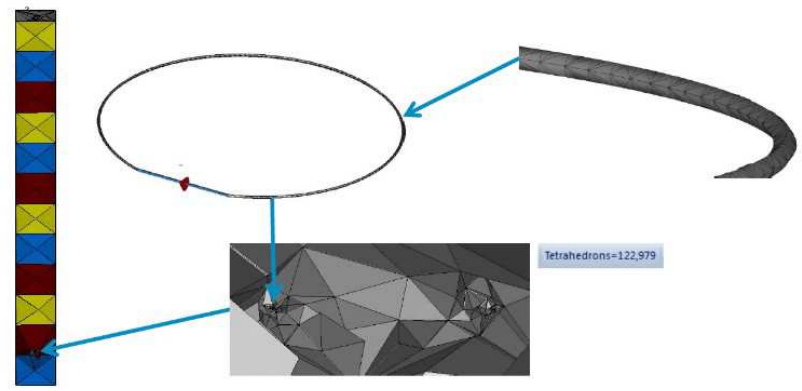

Fig. 8: Details of tetrahedral meshing used by CST software.

electrical plans, magnetic plans, free space, conductive wall, wall with regular features and unit cell. In Figure 9, the open boundaries option is selected on all the walls.

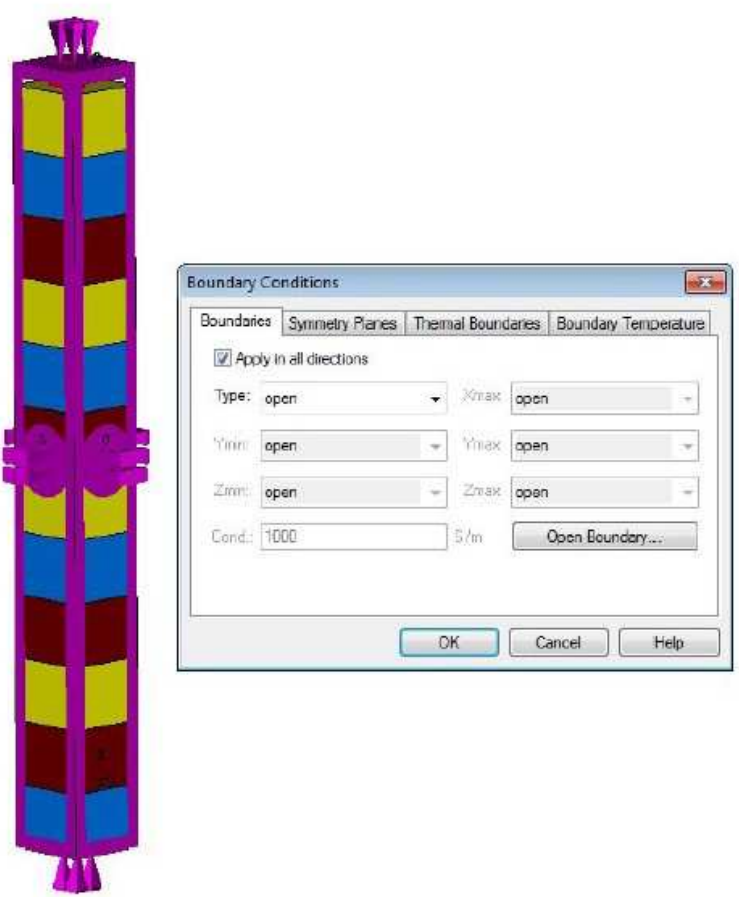

Fig. 9: Detail of computational setup to specify boundary conditions for the finite meshing.

Sensors can measure not only the field level, but also, the user can measure other magnitudes, such as voltages and cur- 
rents levels, S-parameters, Z and Y matrices, VSWR (voltage standing wave ratio), among others. Typically, logarithmic, polar, magnitude and phase 2D/3D graphs are responses of such measures. Some measurements may be displayed as animated images as a function of space and time, or as a vector set that indicates the field direction as a function of space or time. Figure 10 illustrates the magnetic field direction varying in space.

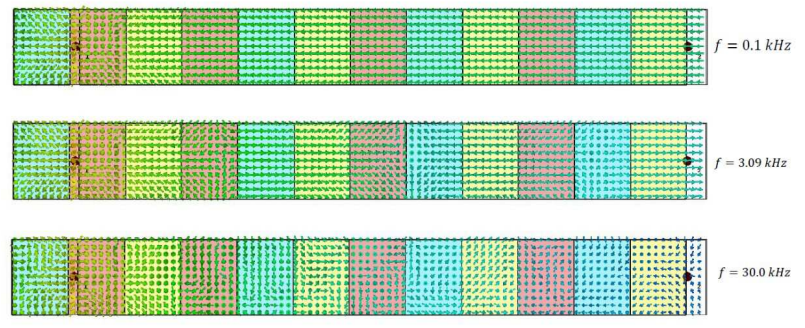

Fig. 10: Magnetic field in the substrates.

Using the same conditions that generated the theoretical curves shown in Section III, we performed computer simulations results on a non-stratified medium. Parameters are: loop radius of $1 \mathrm{~m}$, maximum range of $327 \mathrm{~m}$, soil conductivity $0.08792 \mathrm{~S} / \mathrm{m}$, and coaxial configuration. Figures 11 and 12 illustrate the field intensity attenuation and the channel transfer function, considering suitable theoretical models compared with certain normalized frequency value. Other simulations show that the accuracy extends to higher frequencies when increasing the width of the environment that represents the soil.

\section{B. Link Simulation}

This subsection presents simulation results of a TTE communication link. Figure 13 shows the system block diagram. The purpose of the simulation is to investigate the influence of the TTE channel and atmospheric noise in this system.

Regarding the channel model, we used the CIM scenario with coaxial aligned loop antennas, and we apply the transfer function defined by (11). We also consider that the centres of the loop antennas are separated by a vertical distance $r=$ $200 \mathrm{~m}$ and the soil has conductivity $\sigma=10^{-2} \mathrm{~S} / \mathrm{m}$. Figure 14 shows the magnitude of the channel transfer function for these parameters.

The channel is band-pass and frequency-selective, so that intersymbol interference (ISI) occurs. We modulate a carrier in the optimal frequency $f_{\text {opt }} \approx 5 \mathrm{KHz}$, and use binary phase shift keying (BPSK) modulation, with square-root raised cosine (RCOS) pulse with roll-off factor $\beta=0.5$. The transmission rate is $R=6 \mathrm{kbit} / \mathrm{s}$, so that the transmitted signal occupies a band of $B=9 \mathrm{kHz}$, centred at $f_{\text {opt }}$, and it is corrupted by atmospheric VLF non-Gaussian noise.

The detection and demodulation algorithms of a conventional communication system usually assume additive white Gaussian noise (AWGN). As seen in the previous section, the atmospheric noise in the VLF band consists of a Gaussian component and a non-Gaussian component. Therefore, it is interesting to investigate the performance of conventional

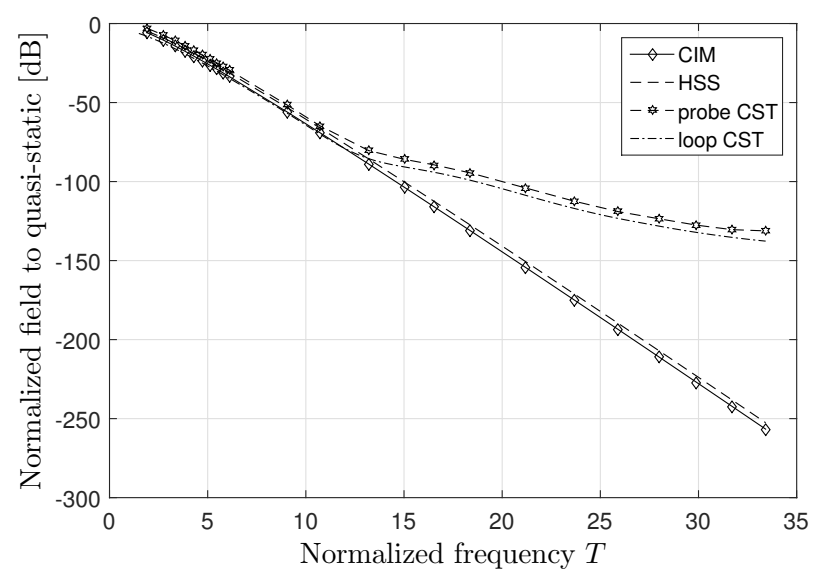

Fig. 11: $\boldsymbol{H}$ field intensity. Comparison of theoretical and simulated models (CST) for CIM and HSS scenarios.

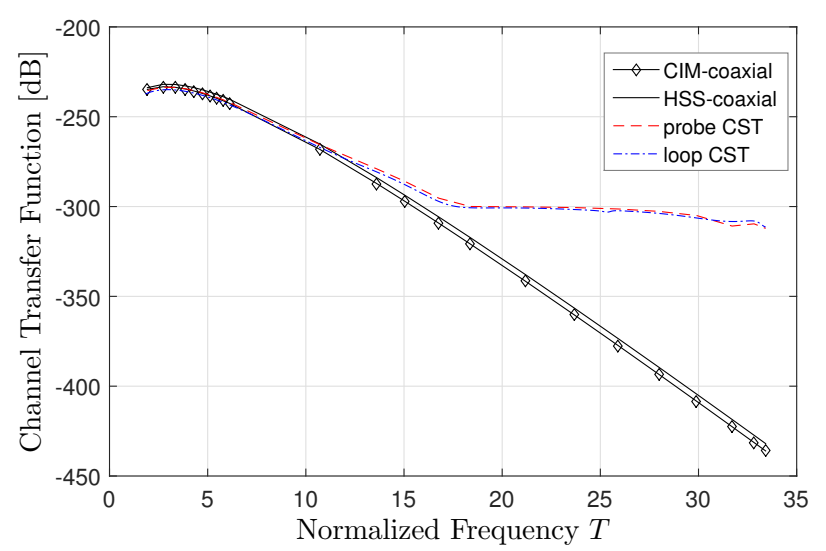

Fig. 12: Channel transfer function. Comparison of theoretical and simulated models (CST) for coaxial configuration, CIM and HSS scenarios.

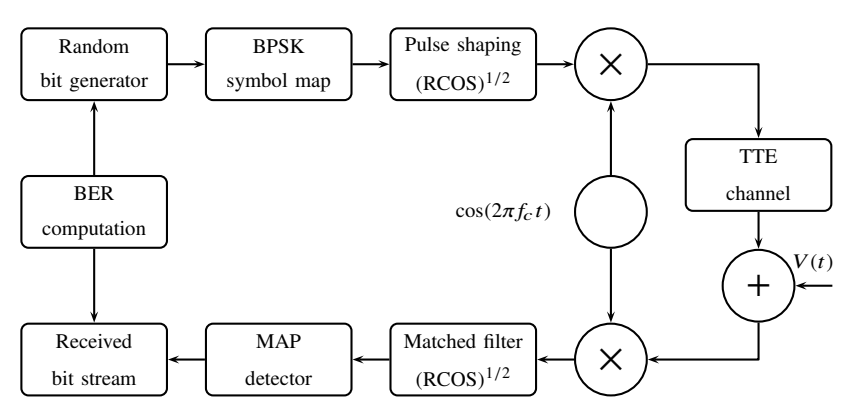

Fig. 13: Block diagram of point-to-point, link-level TTE communication system

AWGN detectors when the noise is non-Gaussian. In addition, as discussed in previous sections, the TTE channel distorts the signal, which degrades the communication system performance, if no equalizer is employed.

Figure 15 shows how the TTE channel, described by the transfer function, Fr, and the atmospheric noise, described by 


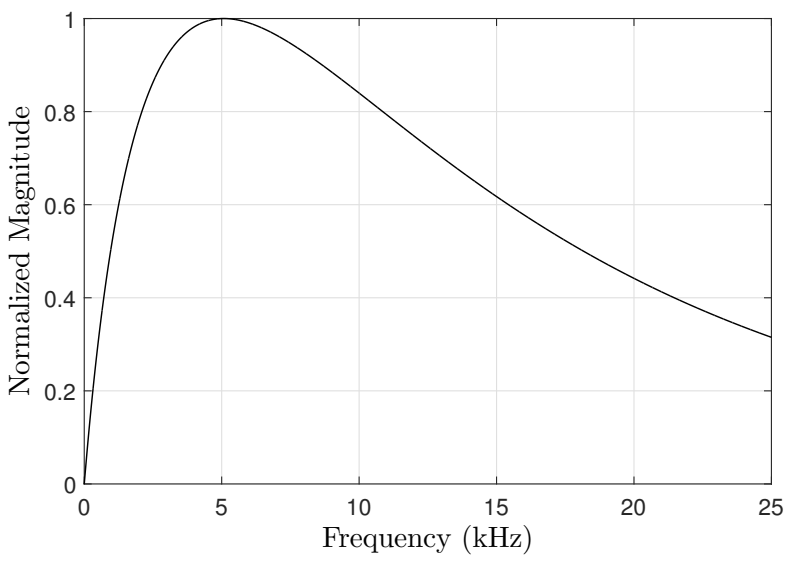

Fig. 14: Magnitude of the channel transfer function for linklevel simulations, with $\sigma=0.01 \mathrm{~S} / \mathrm{m}$ and $r=200 \mathrm{~m}$.

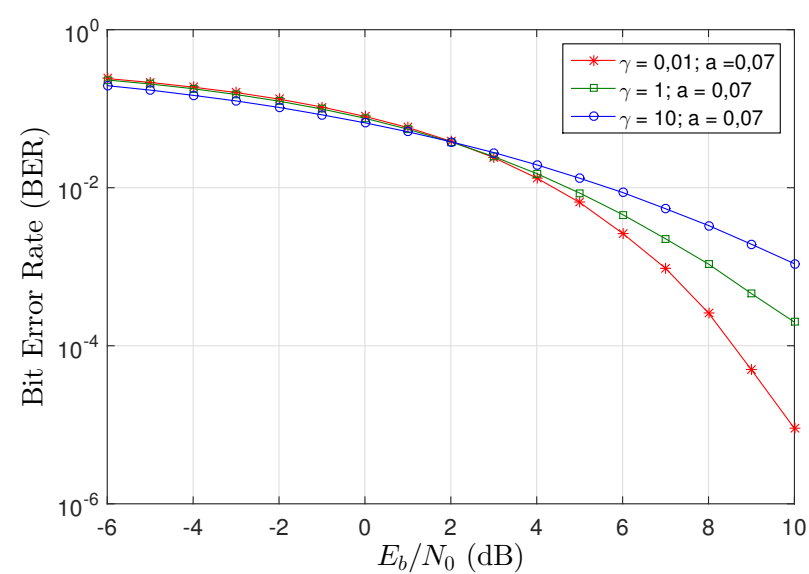

Fig. 15: Bit error rate (BER) for the described TTE communication system, with different levels of impulsive noise.

the Field-Lewinstein model, can affect the performance of a TTE communication system. The curves in Figure 15 show bit error rates for different values of impulsivity, $\gamma$. As seen in Section IV, $\gamma^{2}$ is the energy ratio between the impulsive noise component and the Gaussian component. By modifying it, we control the amount of the impulsive noise component energy.

When $\gamma=0.01$, the noise is practically Gaussian. When $\gamma=1$, Gaussian and impulsive noise component have the same energy amount. Finally, when $\gamma=10$, the Gaussian component energy is 100 times smaller than the impulsive component energy, which becomes dominant. Note that the performance worsens considerably for larger values of impulsive noise energy. With this simulation, it is clear that we cannot ignore the impulsive noise in the receiver design of a TTE communication system.

\section{Medium Access Protocols and Applications}

The literature on medium access (MAC) protocols for TTE communications is practically non-existent, making this one of the major challenges for the research in this area. After taking into account the unique aspects of the transmission medium and the point-to-point link, here we considered some aspects for the specification of a suitable datagram and medium access control protocol for TTE networks.

A first aspect to consider is the restrictions imposed by the communication channel. Due to low transmission rates, we should think of a robust protocol with a minimum amount of information bits aimed to provide data control. Furthermore, as the channel bandwidth, optimal frequency and attenuation depend on the depth and relative angle of the underground terminals, the protocol should consider the transmission in a variable-length band, with different carrier frequencies, and with variable a transmission rate. The need of flexibility also appears when one consider the fact that TTE communications are not only related to disaster situations, but also for mining automation applications, so it is desirable not only to cope with physical aspects of the channel, but also with the possibility to deal with different types of services. For example, voice applications require less control data to ensure message integrity than remote detonation.

Another aspect, given the low transmission rate, is the need of using compressed messages. However, in this case, any error caused by the transmission channel will result in many errors in the decoding process. In the case of uncompressed messages, the error will be local and can be easily removed with an appropriate signal processing technique for that message type. So, the use of error correcting codes for compressed messages is necessary, and a study must be made to assess which codecs and error correcting codes should be used to optimize the packet size.

Two different scenarios may be considered: first, rescue people buried by landslides. In this scenario, transmission of voice, text and eventually photos and video are of utmost importance for the rescue teams, and source compression techniques for voice and image are essential for communications viability. In a second scenario, regarding detonation and automation of mining equipment, transmission rates may be lower, but the integrity of the communication becomes crucial for successful applications. Here, the use of advanced channel coding techniques and specification of efficient network protocols must be addressed.

Still related to the physical channel, transmission is more difficult in the uplink. This happens because the atmospheric noise is more intense on the mine surface, but also because the transmitting equipment inside the mine is likely to be powered by batteries, and has several power restrictions for safety reasons. One possible solution to this problem is the use of repeater nodes in the mine.

Regarding the need of multiple access to the channel, it is necessary to consider whether the MAC protocol will be random or scheduled. If access is random, it can take advantage of the variable transmission rate among users to improve the performance of classic protocols such as Aloha, that is premised on the equal transmission rate for all users. If access is scheduled, the use of FDMA and CDMA is hampered by limited bandwidth, and TDMA use tends to be more probable. Therefore, there is a need for further study on techniques that allow the synchronization among nodes. 


\section{CONCLUSION}

Through-The-Earth communication imposes severe restrictions on the communication system design. The use of signals at low frequencies is essential to make the attenuation provided by the ground not prohibitive, considering the typical depths for underground mines. The presence of non-Gaussian noise and possible power limited transmissions prevent, in a first analysis, the use of higher-order digital modulations with greater spectral efficiency.

Despite these factors, TTE systems are an alternative to wired solutions or infrastructured wireless networks for communication in mines. In this tutorial we have presented a model of a TTE channel, performed link-level simulations based on this model, and addressed some issues regarding higher-layer protocols.

Mining is a very important industry branch, and reliable communication links are a factor to increase its efficiency. By means of this tutorial we hope to have raised awareness to this promising communications technique, that is likely to gain importance in the near future, and, thus, motivate further studies in this area.

\section{ACKNOWLEDGEMENTS}

The authors would like to express their thanks to Vale Technology Institute (ITV) for the financial support of this work.

\section{REFERENCES}

[1] T. D. Barkand, N. W. Damiano, and W. Shumaker, "Through-the-earth, two-way, mine emergency, voice communication systems," in Industry Applications Conference, 2006. 41st IAS Annual Meeting. Conference Record of the 2006 IEEE, vol. 2. IEEE, 2006, pp. 955-958.

[2] F. H. Raab and I. R. Joughin, "Signal processing for through-the-earth radio communication," Communications, IEEE Transactions on, vol. 43, no. 12, pp. 2995-3003, 1995, doi:10.1109/26.477502.

[3] M. R. Yenchek, G. T. Homce, N. W. Damiano, and J. R. Srednicki, "Niosh-sponsored research in through-the-earth communications for mines: a status report," Industry Applications, IEEE Transactions on, vol. 48, no. 5, pp. 1700-1707, 2012, doi:10.1109/TIA.2012.2209853.

[4] D. N. de Produção Mineral, "Código de mineração - Capítulo I das Disposições Preliminares," http://www.dnpm-pe.gov.br/Legisla/cm_01.htm, Accessed: April 22, 2015.

[5] S. Yarkan, S. Güzelgöz, H. Arslan, and R. Murphy, "Underground mine communications: A survey," Communications Surveys Tutorials, IEEE, vol. 11, no. 3, pp. 125-142, rd 2009, doi:10.1109/SURV.2009.090309.

[6] A. M. T. (UNDERGROUND), "Magneto mine telephones," http://www.britishtelephones.com/ atm/atmtel6.htm, 10 2012, Accessed: April 22, 2015

[7] L. Bandyopadhyay, S. Chaulya, and P. Mishra, "Wireless communication in underground mines," RFID-Based Sens. Netw, 2010.

[8] N. I. for Occupational Safety and Health, "Tutorial on wireless communication and electronic tracking part 1: Technology overview," http://www.msha.gov/techsupp/PEDLocating/ WirelessCommandTrack2009.pdf, 4 2010, Accessed: April 22, 2015.

[9] A. Patri, A. Nayak, and S. Jayanthu, "Wireless communication systems for underground mines-a critical appraisal," International Journal of Engineering Trends and Technology (IJETT), vol. 4, no. 7, pp. 3149$3153,2013$.

[10] G. Jing and W. Qianping, "Application of hybrid routing protocol for mine wsn," in Environmental Science and Information Application Technology (ESIAT), 2010 International Conference on, vol. 3, July 2010, pp. 353-355.

[11] B. Chetan, P. Deshpande, B. Gireesh Hegde, S. Srinivas, and G. Narendra Kumar, "Analysis of dsdv amp; aodv for disaster management system in coal mines," in Wireless Communications, Networking and Mobile Computing (WiCOM), 2011 7th International Conference on, Sept 2011, pp. 1-4.
[12] S. Lei, X. Zhao, Z. Wenyan, and C. Xicheng, "Model of ad hoc networks for rescuing in mine," in Networks Security Wireless Communications and Trusted Computing (NSWCTC), 2010 Second International Conference on, vol. 1, April 2010, pp. 210-213.

[13] D. Wu, R. Li, and L. Bao, "A holistic routing protocol design in underground wireless sensor networks," in Mobile Ad-hoc and Sensor Networks, 2008. MSN 2008. The 4th International Conference on, Dec 2008, pp. 187-194.

[14] Z. Zheng and S. Hu, "Research challenges involving cross-layered communication protocol design for underground wsns," in Anticounterfeiting, Security and Identification, 2008. ASID 2008. 2nd International Conference on, Aug 2008, pp. 120-123.

[15] PIMM, "Pilot for Industrial Mobile Communication in Mining," Available: https://www.sics.se/projects/pimm, (2016, May 20).

[16] G. S. et al., "To the rescue!" World Coal, 122010.

[17] E. News, "New through earth communication system for coal mining," Endeavour Magazine, 112014.

[18] D. Gibson, "Channel characterisation and system design for sub-surface communications," Ph.D. dissertation, School of Electronic and Electrical Engineering, 22003.

[19] D. B. Starkey, "Electromagnetic transmission and detection at deep depths," Sandia Laboratories Report SLL-73-5278, 1973.

[20] D. J. Griffiths, Introduction to Electrodynamics, 3rd ed. Prentice Hall, 1999.

[21] J. R. Wait, "Electromagnetic induction technique for locating a buried source," Geoscience Electronics, IEEE Transactions on, vol. 9, no. 2, pp. 95-98, April 1971, doi:10.1109/TGE.1971.271473.

[22] J. R. Wait and K. Spies, "Subsurface electromagnetic fields of a circular loop of current located above ground," Antennas and Propagation, IEEE Transactions on, vol. 20, no. 4, pp. 520-522, Jul 1972, doi:10.1109/TAP.1972.1140232.

[23] J. Durkin, "Surface vertical magnetic field produced by a finite loop buried in an earth containing a thin conducting sheet," Radio Science, vol. 32, no. 1, pp. 19-23, 1997, doi:10.1029/96RS03202.

[24] P. Johannessen, "Automatic tuning of high-q antenna for vlf fsk transmission," Communications Systems, IEEE Transactions on, vol. 12, no. 1, pp. 110-115, March 1964, doi:10.1109/TCOM.1964.1088894.

[25] H. Wheeler, "Small antennas," Antennas and Propagation, IEEE Transactions on, vol. 23, no. 4, pp. 462-469, Jul 1975, doi:10.1109/TAP.1975.1141115.

[26] M. Rhodes and B. Hyland, "Antenna formed of multiple resonant loops," apr 24 2012, uS Patent 8,164,530 B2. [Online]. Available: http://www.google.com/patents/US8164530

[27] F. H. Raab, "Noise model for low-frequency through-the-earth communication," Radio Science, vol. 45, no. 6, 2010, doi:10.1029/2010RS004378.

[28] E. Field and M. Lewinstein, "Amplitude-probability distribution model for vlf/elf atmospheric noise," Communications, IEEE Transactions on, vol. 26, no. 1, pp. 83-87, 1978, doi:10.1109/TCOM.1978.1093971.

[29] F. H. Raab, "Extraction of vlf-noise parameters," in Military Communications Conference, 1992. MILCOM'92, Conference Record. Communications-Fusing Command, Control and Intelligence., IEEE. IEEE, 1992, pp. 1040-1045.

[30] CST, "Computer Simulation Technology," Available: http://www.cst.com, (2015, July 1).

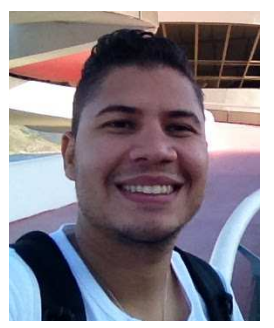

Josua Daniel Peña Carreño was born in Caracas, Venezuela, in 1985. He obtained the B.S. degree in Electrical Engineering at the Central University of Venezuela, Venezuela and M.Sc. in Electrical Engineering at the University of Brasília, Brazil. He is pursuing the $\mathrm{PhD}$. degree in Electrical Engineering at University of Brasília, Brazil. His research interests are antennas and propagation and wireless communications.

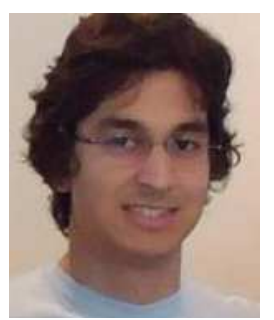

Lucas Sousa e Silva was born in São Paulo, Brazil, in 1991. He obtained the B.S. degree in Electrical Engineering from the University of Brasília, Brazil. He is pursuing the M.Sc. degree in Electrical Engineering at the University of Brasília, Brazil. His research interests are communication theory, information theory and wireless communications. 


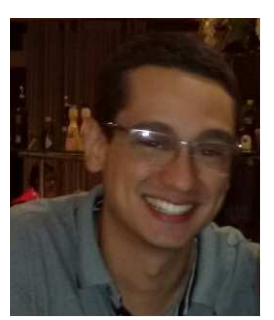

Sávio Oliveira de Almeida Neves was born in Brasília, Brazil, in 1992. He obtained the B.S. degree in Communication Networks Engineering from University of Brasília, Brazil. He is pursuing the M.Sc. degree in Electrical Engineering at University of Brasília, Brazil. His research interests are communication theory, information theory and wireless communications.

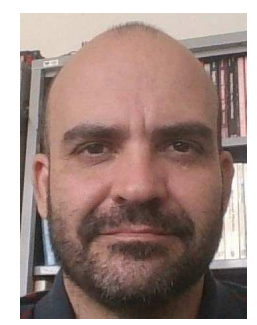

Leonardo Aguayo was born in São Paulo, Brazil, in 1971. He received the B.S. and M.Sc. degrees in electrical engineering from the Polytechnic School of the University of São Paulo, Brazil, in 1994 and 1999, and the Ph.D. in teleinformatics from the Federal University of Ceará (UFC), Brazil, in 2008. $\mathrm{He}$ also holds an M.B.A. degree from the Brazilian Institute of Capital Markets (IBMEC). He worked in Brazil as an RF engineer and consultant, as undergraduate Professor at the University of Fortaleza, and as researcher for the Department of Teleinformatics of Federal University of Ceará. After five years of R\&D activities at Nokia Institute of Technology (INdT) in Brasília, since 2011 he has been with the Electronics Engineering department at University of Brasília, Gama campus. His main research interests are time series processing, wireless communication systems and neural networks.

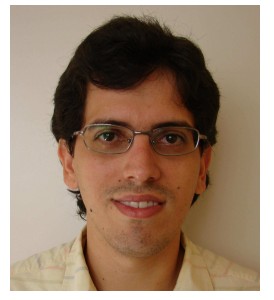

Adoniran Judson Braga was born in Belém, Brazil, in 1975. He received the B.S. and M.Sc. degrees in electrical engineering from the Federal University of Pará, Belém, Brazil, in 1999 and 2001, respectively, and the Diplôme de Doctorat in telecommunications and electronics from Telecom ParisTech, Paris, France, in 2006. He also conducted studies as postdoctoral researcher at the National Institute of Standards and Technology (NIST), Maryland, USA, from 2007 to 2008 and at the École Nationale Supérieure de Techniques Avancées (ENSTA), Paris, France, from 2008 to 2009. Since 2009 he has been with the Department of Electrical Engineering at the University of Brasília. He has experience in electrical engineering with emphasis on communication systems, wave propagation, antennas and array processing. He has laboratory experience in implementing communication systems, radars and channel sounders.

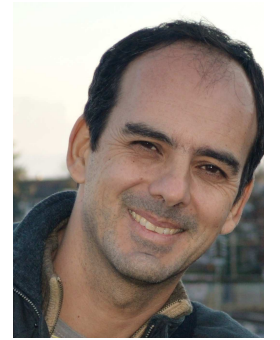

André Noll Barreto graduated in Electrical Engineering at PUC-Rio in 1994, where he also earned a master's degree in 1996. In 2001 he completed his $\mathrm{PhD}$ at the TU Dresden, Germany, with highest degree (summa cum laude). He is co-founder of Ektrum and currently part-time professor at the University of Brasília (UnB). He has previously worked as a research assistant at TU-Dresden, as post-doctoral fellow at the IBM Research Center in Zurich, as a planning Engineer at Claro, Rio de Janeiro, and as a research specialist at Nokia Institute of Technology (INdT), Brasília. He is the author of four international patents and numerous articles in journals and international conferences. His current research areas of interest are in wireless communication systems, particularly 5G and IoT, and through-the-earth communications. He has experience in technologies such as CDMA, GSM, EGPRS, IEEE802.11, LTE and 5G, in the development of link- and system-level simulators, and in modulation schemes for optical communications. He was the general chair of Brazilian Telecommunications Symposium in 2012 and chair of the Centro-Norte Brasil IEEE Section in 2011/2012.

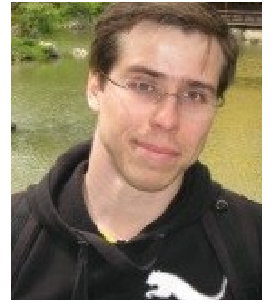

Luis Guilherme Uzeda Garcia was born Curitiba, Brazil, in 1979. In 2015, he spent a semester at the Massachusetts Institute of Technology (MIT) working as an International Faculty Fellow. He holds a $\mathrm{Ph} . \mathrm{D}$. degree in wireless communications from Aalborg University (AAU), and M.Sc. E.E. and B.Sc. degrees in electronics and computer engineering from the Federal University of Rio de Janeiro (UFRJ/COPPE). Besides through-the-earth communications, his current research interests include the role of wireless systems in cyberphysical systems, cybersecurity, machine learning and smart industries. Since 2013 he has been working at the Vale Institute of Technology (ITV), a nonprofit R\&D institute founded by Vale, the world's largest iron ore producer. 